\title{
Benzil fluorescence and phosphorescence emissions: a pertinent probe for the kinematic behaviour and microheterogeneity of supercritical $\mathrm{CO}_{2}$
}

\author{
Nitin Chattopadhyay ${ }^{\mathrm{a}, \mathrm{b}, *}$, Carlos Serpa ${ }^{\mathrm{a}}$, M. Isilda Silva ${ }^{\mathrm{a}}$, \\ Luis G. Arnaut ${ }^{\text {a }}$, Sebastião J. Formosinho ${ }^{\text {a }}$ \\ a Department of Chemistry, Coimbra University, P-3004-535, Coimbra, Portugal \\ b Department of Chemistry, Jadavpur University, Calcutta 700 032, India
}

Received 2 August 2001; in final form 3 September 2001

\begin{abstract}
The relative intensity (RI) of the phosphorescence and fluorescence from the relaxed trans-planar geometry of benzil has been studied as a function of pressure and temperature in supercritical carbon dioxide $\left(\mathrm{SC}-\mathrm{CO}_{2}\right)$. The nature of the variation of RI with pressure and temperature is similar to that of the kinematic viscosity (KV) with the two said parameters. The experimental results have been interpreted in terms of microheterogeneity of the supercritical fluid (SCF). (C) 2001 Elsevier Science B.V. All rights reserved.
\end{abstract}

\section{Introduction}

Supercritical fluids (SCF), with their unusual properties, have received widespread attention in diverse fields [1-6]. One of the most important properties of SCF is the local density effect on solute molecules [7-9]. The density of a SCF is between that of a gas and a normal liquid, and can be easily tuned with a change in pressure under isothermal conditions. Consequently, medium properties can be continuously varied without a change of solvent.

\footnotetext{
${ }^{*}$ Corresponding author. Fax: +351-239-827-703 and +91-33473-4266.

E-mail address: pcnitin@yahoo.com (N. Chattopadhyay).
}

Amongst a wide variety of techniques [10-13] employed to characterise SCF properties, the high sensitivity of the techniques of fluorescence and phosphorescence makes them particularly useful. The fluorescence and phosphorescence properties of benzil and similar vicinal bi-carbonyl compounds have long been the subject of immense research [14-18] principally because of the interesting geometric relaxation of the fluorophore molecules in the photoexcited states, coupled with reasonably intense room temperature phosphorescence in fluid phases. Fluorescence and phosphorescence have been observed from both the unrelaxed (skew) and the relaxed (trans-planar) conformations of the systems. The emissions with maxima at around 500 and $560 \mathrm{~nm}$ at ambient temperatures in liquid solutions have been as- 
signed as the fluorescence and phosphorescence respectively from the relaxed trans-planar structure of benzil $[15,18]$. Being trans-planar, the relaxed configuration of benzil has a zero dipole moment. Consequently, both the 500 and $560 \mathrm{~nm}$ emissions do not show any solvent shift [18].

It is known that the phosphorescence yield is highly sensitive to the fluidity of the environment. In contrast, the fluorescence yield is much less sensitive to the same factor. Thus, the relative intensity (RI) of the two emissions can be used as a tool to study the density fluctuations in SCFs. In the present Letter, we report our endeavour to exploit this RI parameter to study the kinematic behaviour of supercritical $\mathrm{CO}_{2}$. A discussion on the microheterogeneous structure of the SCF will justify the experimental observations.

\section{Experimental}

Benzil (Aldrich) was purified by sublimation followed by recrystallisation from ethanol. For the preparation of the mother solution, spectrophotometric grade diethylether (Aldrich) was used as received.

The emission studies were performed in a Spex Fluorolog-3 spectrofluorimeter. The individual components were extracted by decomposing the overlapping emissions. The spectroscopic studies were carried out using a home-made stainless steel high pressure optical cell with four sapphire windows. The temperature within the cell was controlled and measured using an OMEGA CN77000 series temperature controller $\left( \pm 0.5^{\circ} \mathrm{C}\right)$ and the pressure inside the cell was measured with a OMEGA DP20 digital pressure indicator $( \pm 0.25 \%)$. Fig. 1 gives the schematic design of our SCF apparatus.

For the emission experiments the sample was prepared in the following way. A solution of benzil $\left(2 \times 10^{-4} \mathrm{M}\right)$ was prepared in diethylether and was loaded in the clean SCF cell. The solvent ether was then completely evaporated. The cell was evacuated and filled with $\mathrm{CO}_{2}$ after thermostating at the experimental temperature.

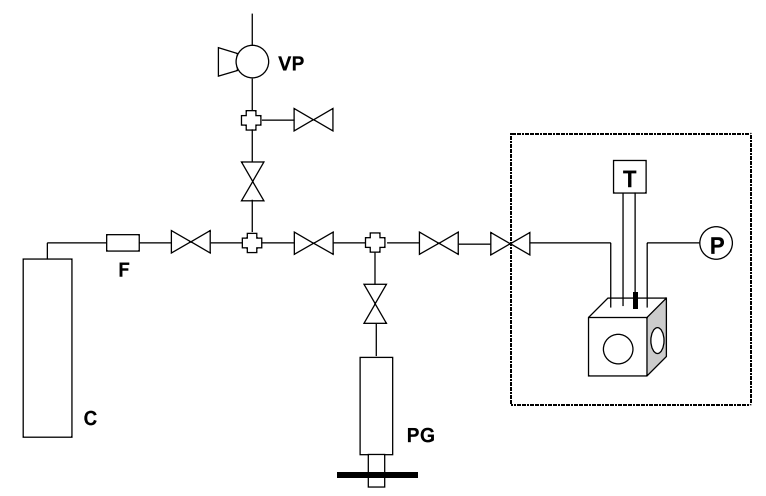

Fig. 1. Schematic diagram of the SCF apparatus: $\mathrm{C}, \mathrm{CO}_{2}$ cylinder; F, filter; VP, vacuum pump; PG, pressure generator; $T$, Temperature controller; $P$, Pressure indicator.

\section{Results and discussion}

Photoluminescence of benzil has been studied in supercritical carbon dioxide. Because the collision of the excited molecule with the solvent the phosphorescence intensity is reduced very efficiently due to the enhanced non-radiative deactivation of the triplet state. Phosphorescence is, thus, much more sensitive to the fluidity of the environment compared to that of the fluorescence. Hence to minimise the instrumental response dependence on the experimental data, we have studied the variation of the relative intensity (RI) of the 560 (phosphorescence) and $500 \mathrm{~nm}$ (fluorescence) emissions of benzil as a function of pressure and temperature of the $\mathrm{SC}-\mathrm{CO}_{2}$. The RI shows a dependence on both the pressure and the temperature of the SCF.

Fig. 2 represents the normalised emission spectra of benzil and depicts the change in the RI with a change in the SCF pressure at $308 \mathrm{~K}$. At any particular temperature over the range studied, with an increase in pressure, intensity of the 560 $\mathrm{nm}$ emission first decreases and then increases again, giving rise to a minimum (Fig. 3). The slope of the first part is, however, greater than that of the latter part. With an increase in the temperature, the well becomes shallower and the minimum $\left(P_{\min }\right)$ shifts towards higher pressure (Fig. 3). The isotherms reproduce exactly, the kinematic viscosity (KV) isotherms when plotted against pres- 


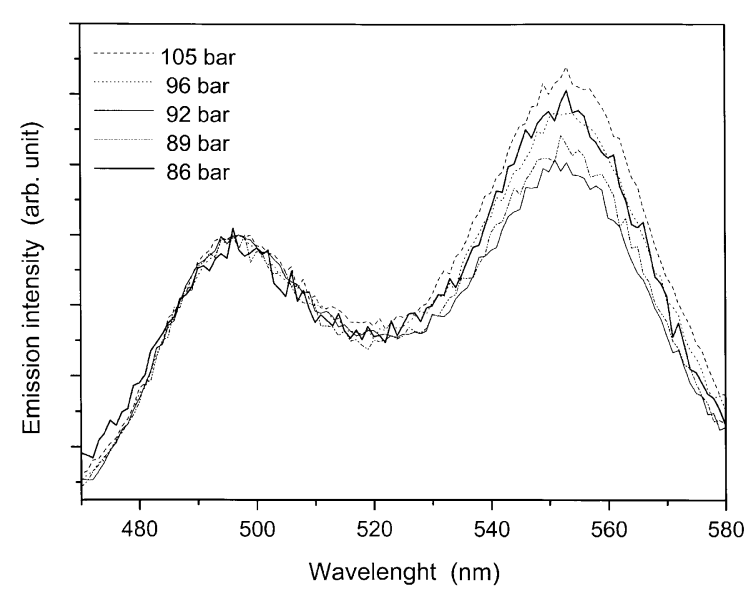

Fig. 2. Emission spectra of benzil in $\mathrm{SC}-\mathrm{CO}_{2}$ at $308 \mathrm{~K}$.

sure [19]. The effect of temperature on the RI parameter also follows the same pattern as that of the KV. Thus, it is revealed that the variation of $\mathrm{RI}$ follows the $\mathrm{KV}$ of the supercritical $\mathrm{CO}_{2}$ environment. The published $\mathrm{KV}$ isotherms have, however, been generated from calculation [19]. To our knowledge, this is the first experimental report employing the emission technique, involving phosphorescence and fluorescence together, to probe the KV of a SCF.

The equivalence of the KV with our RI parameter is not unexpected as the former represents the fluidity of the SCF environment on which the latter largely depends. It has been observed, although only over a short range of temperature, that the minimum $\left(P_{\min }\right)$ of the plot of RI against pressure follows a linear relationship with the temperature (Fig. 4). The linearity relation of our data agrees extremely well with that of Bartle et al. [19] again confirming that the RI of benzil can act as an useful probe for the fluidity of the SCF environment.

The variation of the RI with pressure and temperature can be interpreted considering a molecular view of the $\mathrm{SC}-\mathrm{CO}_{2}$ system. Two mechanisms may lead to a decrease in the phosphorescence emission at an experimental temperature: an intramolecular path, where some geometric relaxation within the fluorophore system provides a channel to leak the excess energy of the triplet state and, an intermolecular path, where
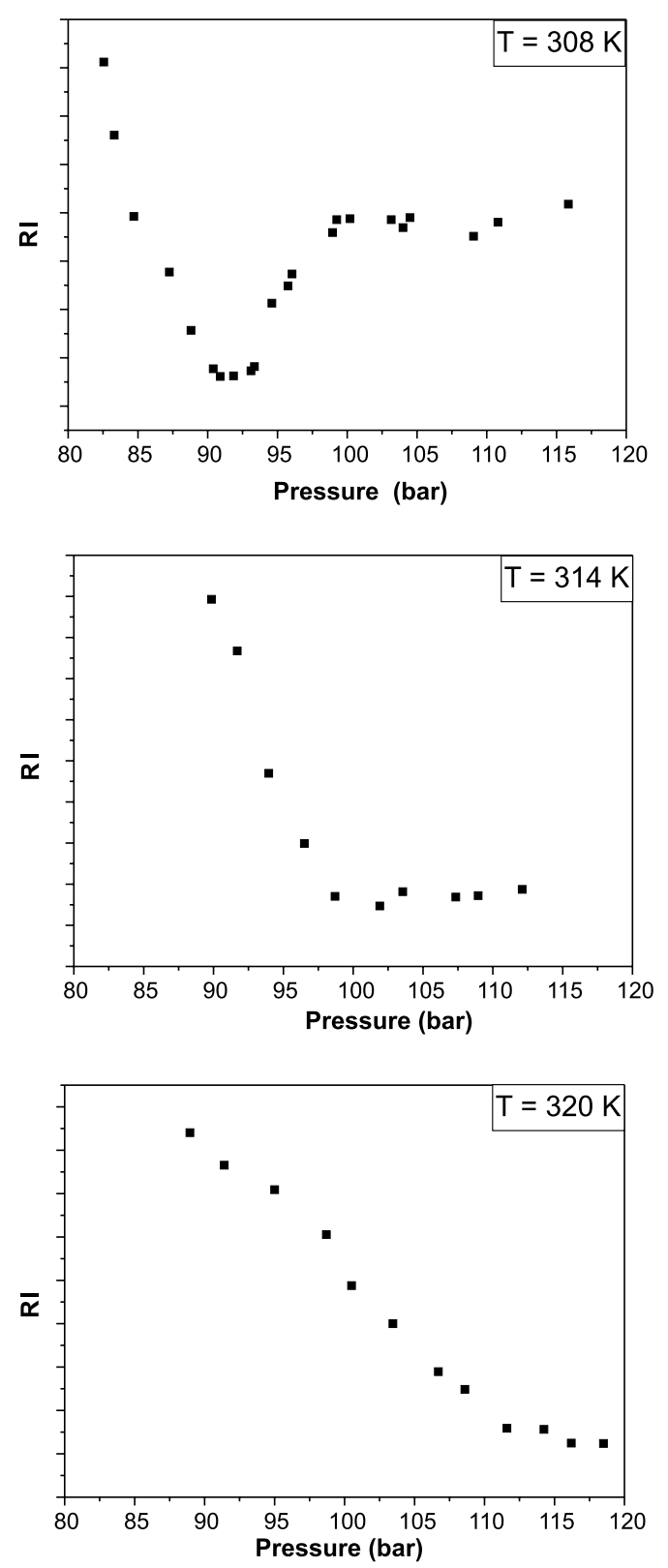

Fig. 3. Plot of RI of benzil in $\mathrm{SC}-\mathrm{CO}_{2}$ as a function of pressure at different temperatures.

the collisional quenching of the fluorophore with the solvent molecules promotes the radiationless decay of the triplet state. Since both the emissions $(500$ and $560 \mathrm{~nm})$ considered here originate from the most relaxed state (trans-planar) of benzil, 


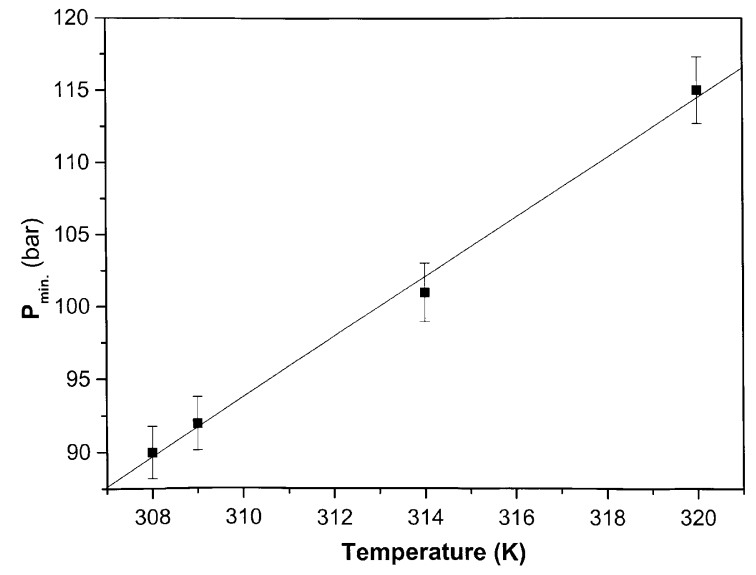

Fig. 4. Variation of $P_{\min }$ against temperature.

quenching of the phosphorescence by geometric relaxation does not appear likely in the present case. The differential effect of the polarity of the $\mathrm{SCF}$ on the two emissions is also ruled out from the fact that both the emissions originate from the trans-planar geometry of benzil, which has a zero dipole moment [18]. Thus, it is reasonable to consider collisional quenching to be responsible for a variation of the RI with pressure and/or temperature.

The observed variation of RI with pressure and temperature can be explained by considering the local density effect in the SCF at various pressures. In the low pressure region, the local density of the solvent molecules is lower leading to fewer solvent molecules that can collide with benzil to deactivate the triplet. With an increase in the pressure the solvent molecules come closer to the fluorophor. This leads to an enhanced short-range solute-solvent interaction, corresponding to a process of saturation of the inner solvation shell resulting in a marked decrease in the phosphorescence intensity. Thus, before saturation, addition of the solvent molecules to the inner solvent shell causes increased non-radiative deactivation of the triplet resulting in strong pressure dependence. The inner shell solvation can approximately be described in terms of the Langmuir equilibrium model [20]. The minimum in the plot of RI against pressure is reached when the inner solvent shell is saturated probably with a monolayer of solvent molecules.
After the formation of the saturated inner solvent shell, further increase in the pressure induces some rigidity in the microenvironment around the fluorophore either by reducing the size of the shell due to the proximity of other similar units or by increasing the size of the clusters through the formation of secondary solvent shell and hence reducing the flexibility of the units. Since the probe molecule is guarded within the inner shell solvent molecules, it is protected from the collision with other solvent molecules of the secondary shell in the cluster, and the increase in cluster size is ineffective in causing further deactivation of the triplet. In contrast, the rigidity of the microenvironment leads to an increase in the phosphorescence yield. Of course, this increase in the rigidity of the microenvironment is only marginal, giving only a little enhancement in the phosphorescence yield.

Thus, we consider that the supercritical $\mathrm{CO}_{2}$ makes a microheterogeneous environment, rather than a homogeneous one, around the probe. The concept of microheterogeneity of the supercritical environments has already been adopted to explain various observations in different SCFs including $\mathrm{CO}_{2}$ [2,21-24]. With an increase in the temperature, the kinetic energy of the solvent molecules increases, thereby requiring a higher pressure to saturate the inner shell. This is consistent with the observed shift of the $P_{\min }$ towards higher pressure at elevated temperature. Furthermore, the increased molecular velocity at high temperature makes the boundary of this shell less precise, leading to the flattening of the well, as is observed.

\section{Conclusion}

It has been shown for the first time that the relative intensities (RI) of the phosphorescence and fluorescence from the relaxed geometry of excited benzil can be utilised to monitor the kinematic behaviour of the supercritical fluids. The variation of the RI with pressure and temperature has been studied and explained from the molecular viewpoint of the fluid. It has been proposed that supercritical $\mathrm{CO}_{2}$ forms a microheterogeneous 
environment around the probe rather than a homogeneous one.

\section{Acknowledgements}

Financial supports from PRAXIS/PCEX/QUI/ 0108/96 (European Union) and Fundação para a Ciência e a Tecnologia (grant BD/18362/98) are gratefully acknowledged. The authors sincerely thank Professor H.D. Burrows for helpful discussions. We thank Luis Baptista for experimental assistance.

\section{References}

[1] T. Clifford, Fundamentals of Supercritical Fluids, Oxford University Press, London, 1999.

[2] S.C. Tucker, Chem. Rev. 99 (1999) 391.

[3] A. Baiker, Chem. Rev. 99 (1999) 453.

[4] A.J. Mesiano, E.J. Beckman, A.J. Russell, Chem. Rev. 99 (1999) 623.

[5] J.L. Kendall, D.A. Canelas, J.L. Young, J.M. DeSimone, Chem. Rev. 99 (1999) 543.

[6] P.E. Savage, Chem. Rev. 99 (1999) 603.

[7] C.A. Eckert, D.H. Ziger, K.P. Johnston, S. Kim, J. Phys. Chem. 90 (1986) 2738.
[8] P.G. Debenedetti, R.S. Mohamed, J. Chem. Phys. 90 (1989) 4528

[9] K.S. Shing, S.T. Chung, J. Phys. Chem. 91 (1987) 1674.

[10] M. Poliakoff, S.M. Howdle, S.G. Kazarian, Angew. Chem. Int. Ed. Engl. 34 (1995) 1275.

[11] A. Kordikowski, D.G. Robertson, M. Poliakoff, T.D. DiNoia, M. McHugh, A. Aguiar-Ricardo, J. Phys. Chem. B 101 (1997) 5353.

[12] T.A. Betts, F.V. Bright, Appl. Spectros. 44 (1990) 1203

[13] M.P. Heitz, F.V. Bright, J. Phys. Chem. 100 (1996) 6889.

[14] S.C. Bera, R.K. Mukherjee, M. Chowdhury, J. Chem. Phys. 51 (1969) 754; S.C. Bera, R.K. Mukherjee, M. Chowdhury, Ind. J. Pure Appl. Phys. 7 (1969) 345.

[15] J.F. Arnett, S.P. McGlynn, J. Phys. Chem. 79 (1975) 626.

[16] D.S. Ray, K. Bhattacharyya, S.C. Bera, M. Chowdhury, Chem. Phys. Lett. 69 (1980) 134.

[17] B.E. Kohler, R.T. Loda, J. Chem. Phys. 74 (1981) 18.

[18] K. Bhattacharyya, M. Chowdhury, J. Photochem. 33 (1986) 61.

[19] K.D. Bartle, T. Boddington, A.A. Clifford, G.F. Shilstone, J. Chromatogr. 471 (1989) 347.

[20] A. Morita, O. Kajimoto, J. Phys. Chem. 94 (1990) 6420.

[21] Y-P. Sun, M.A. Fox, K.P. Johnston, J. Am. Chem. Soc. 114 (1992) 1187.

[22] Y-P. Sun, G. Bennett, K.P. Johnston, M.A. Fox, J. Phys. Chem. 96 (1992) 10001.

[23] Y-P. Sun, C.E. Bunker, N.B. Hamilton, Chem. Phys. Lett. 210 (1993) 111

[24] J.F. Brennecke, D.L. Tomasko, J. Peshkin, C.A. Eckert, Ind. Eng. Chem. Res. 9 (1990) 1682. 